\title{
Linear Pencils of Tropical Plane Curves
}

\author{
Filip Cools
}

Received: 5 May 2011 / Revised: 18 January 2012 / Accepted: 24 January 2012 /

Published online: 16 February 2012

(C) Springer Science+Business Media, LLC 2012

\begin{abstract}
Linear pencils of tropical plane curves are parameterized by tropical lines (i.e. trees) in the space of coefficients. We study pencils of tropical curves with $n$ element support that pass through $n-2$ general points in the plane. Richter-Gebert et al. proved that such trees are compatible with their support set, and they conjectured that every compatible tree can be realized by a point configuration. In this article, we prove this conjecture. Our approach is based on a characterization of the fixed loci of tropical linear pencils.
\end{abstract}

Keywords Tropical geometry $\cdot$ Plane curves $\cdot$ Trees

\section{Introduction}

Let $K=\mathbb{C}\{\{t\}\}$ be the field of Puiseux series (or any other algebraically closed field with a non-trivial valuation), and let $\mathcal{A}=\left\{a_{1}, \ldots, a_{n}\right\}$ be a subset of $\{(r, s, t) \in$ $\left.\left(\mathbb{Z}_{\geq 0}\right)^{3}: r+s+t=d\right\}$ for some integer $d$, with $a_{i}=\left(r_{i}, s_{i}, t_{i}\right)$. We always assume that the convex hull $\operatorname{conv}(\mathcal{A})$ is two-dimensional, hence $n \geq 3$. A polynomial $f$ of the form

$$
\sum_{i \in\{1, \ldots, n\}} k_{i} X^{r_{i}} Y^{s_{i}} Z^{t_{i}} \in K[X, Y, Z]
$$

defines a projective plane curve $V(f)$ of degree $d$ in $\mathbb{P}^{2}(K)$. The tropical polynomial corresponding to $f$ is the piece-wise linear map

$$
F_{c}: \mathbb{R}^{3} \rightarrow \mathbb{R}:(x, y, z) \mapsto \min _{i \in\{1, \ldots, n\}}\left\{c_{i}+r_{i} x+s_{i} y+t_{i} z\right\}
$$

The author is a postdoctoral fellow of the Research Foundation-Flanders (FWO).

F. Cools (凶)

Department of Mathematics, KU Leuven, Celestijnenlaan 200B, 3001 Leuven, Belgium e-mail: Filip.Cools@wis.kuleuven.be 
with $c=\left(c_{1}, \ldots, c_{n}\right)=\left(\operatorname{val}\left(k_{1}\right), \ldots, \operatorname{val}\left(k_{n}\right)\right) \in \mathbb{R}^{n}$, which is obtained by replacing all the operations in $f$ by the tropical operations $\oplus$ and $\otimes($ where $x \oplus y:=\min \{x, y\}$ and $x \otimes y:=x+y$ for all $x, y \in \mathbb{R})$ and all the coefficients by its valuations. Let $\mathcal{T}\left(F_{c}\right)$ be the set of all points $(x, y, z) \in \mathbb{R}^{3}$ for which the minimum $F_{c}(x, y, z)$ is attained at least twice. The Fundamental Theorem in Tropical Geometry (see e.g. [4, 9]) tells us that $\mathcal{T}\left(F_{c}\right)$ is equal to closure in $\mathbb{R}^{3}$ of

$$
\left\{(\operatorname{val}(X), \operatorname{val}(Y), \operatorname{val}(Z)) \mid(X, Y, Z) \in V(f) \cap\left(K^{\times}\right)^{3}\right\} .
$$

Since $\mathcal{T}\left(F_{c}\right)$ is closed under tropical scalar multiplication, i.e.

$$
\lambda \otimes(x, y, z)=(\lambda+x, \lambda+y, \lambda+z) \in \mathcal{T}\left(F_{c}\right) \Longleftrightarrow(x, y, z) \in \mathcal{T}\left(F_{c}\right),
$$

we can identify $\mathcal{T}\left(F_{c}\right)$ with its image in the tropical projective plane $\mathbb{T P}^{2}=$ $\mathbb{R}^{3} / \mathbb{R}(1,1,1)$ and we say that $\mathcal{T}\left(F_{c}\right)$ is a tropical projective plane curve with support set $\mathcal{A}$. Consider the convex hull of the points $\left(r_{i}, s_{i}, t_{i}, c_{i}\right) \in \mathbb{R}^{4}$ with $i \in\{1, \ldots, n\}$. Under the projection to the first three coordinates, the lower faces of this polytope map onto the convex hull $\operatorname{conv}(\mathcal{A})$ of $\mathcal{A}$ and give rise to the regular subdivision $\Delta(c)$ of $\operatorname{conv}(\mathcal{A})$. Note that two elements $a_{i}, a_{j} \in \mathcal{A}$ are connected by a line segment in $\Delta(c)$ if and only if there exists a point $(x, y, z) \in \mathbb{T P}^{2}$ such that

$$
c_{i}+r_{i} x+s_{i} y+t_{i} z=c_{j}+r_{j} x+s_{j} y+t_{j} z<c_{k}+r_{k} x+s_{k} y+t_{k} z
$$

for all $k \in\{1, \ldots, n\} \backslash\{i, j\}$, and that the triangle $T=\operatorname{conv}\left\{a_{i}, a_{j}, a_{k}\right\}$ is a face of $\Delta(c)$ if and only if there exists a point $(x, y, z) \in \mathbb{T P}^{2}$ such that

$c_{i}+r_{i} x+s_{i} y+t_{i} z=c_{j}+r_{j} x+s_{j} y+t_{j} z=c_{k}+r_{k} x+s_{k} y+t_{k} z<c_{\ell}+r_{\ell} x+s_{\ell} y+t_{\ell} z$

for all $\ell \in\{1, \ldots, n\} \backslash\{i, j, k\}$. So we can see that the curve $\mathcal{T}\left(F_{c}\right) \subset \mathbb{T P}^{2}$ is an embedded graph which is dual to $\Delta(c)$. We say that $\Delta(c)$ is maximal with respect to $\mathcal{A}$ if and only if $|T \cap \mathcal{A}|=3$ for each face $T$ of $\Delta(c)$ (in particular, $\Delta(c)$ has to be a triangulation). In case $\mathcal{A}=\operatorname{conv}(\mathcal{A}) \cap \mathbb{Z}^{3}$, the curve $\mathcal{T}\left(F_{c}\right)$ is called smooth if and only if $\Delta(c)$ is a maximal triangulation.

Tropical plane curves going through some fixed points have been studied intensively, mainly because of their applications in enumerative algebraic geometry (see for example [5, 8]). The space of tropical plane quadrics through two or three points is examined in [2]. In this article, we focus on tropical plane curves with support set $\mathcal{A}$ passing through $n-2$ points in general position. In classical algebraic geometry (and over the field $K$ ), the set of such curves is a linear pencil parameterized by a line in the coefficient space. The notion of a linear pencil also makes sense in the tropical setting. Indeed, let $L \subset \mathbb{T P}^{n-1}=\mathbb{R}^{n} / \mathbb{R}(1, \ldots, 1)$ be a tropical line (see [12] for a description of the Grassmannian of tropical lines). Note that $L$ is an $n$-tree where the leaves of $L$ (labeled by $1, \ldots, n$ ) are the points at infinity of the unbounded edges (where the leaf $i$ is lying on the ray with direction $e_{i}$ ) and it satisfies the following property: if an edge of $L$ gives rise to a partition $I$ and $I^{c}=\{1, \ldots, n\} \backslash I$ of the leaf set, then the direction of this edge (towards the leaves in $I$ ) is equal to $e_{I}=\sum_{i \in I} e_{i}$. Each point $c=\left(c_{1}, \ldots, c_{n}\right) \in L$ gives rise to a tropical plane curve $\mathcal{T}\left(F_{c}\right)$ with support $\mathcal{A}$, so we can consider $L$ as the parameter space of a linear pencil, and by abuse 
of notation we will also denote this linear pencil by $L$. Note that $L$ can be seen as the image under the valuation map of a linear pencil of plane curves over $K$.

The fixed locus of the linear pencil $L$ is the set of points $P \in \mathbb{T} P^{2}$ such that each curve in $L$ goes through $P$. Unlike linear pencils over $K$, the fixed locus of $L$ is not determined by the intersection of two different curves of $L$. The following result says that each point $P$ in the fixed locus of $L$ corresponds to some curve of $L$, for which $P$ is a special point.

Proposition 1.1 Let $L \subset \mathbb{T} \mathbb{P}^{n-1}$ be a linear pencil of tropical plane curves with support set $\mathcal{A}=\left\{a_{1}, \ldots, a_{n}\right\}$. Then $P \in \mathbb{T P}^{2}$ is a point of the fixed locus of $L$ if and only if there exists a point $c \in L$ such that one of the following two cases holds:

(1) there exist elements $i, j, k, \ell \in\{1, \ldots, n\}$ such that the pairs of leaves $\{i, j\}$ and $\{k, \ell\}$ belong to different components of $L \backslash\{c\}$ and the minimum of $\left\{c_{i}+a_{i}\right.$. $P\}_{i=1, \ldots, n}$ is attained by the terms corresponding to $i, j, k, \ell$;

(2) there exist elements $i, j, k \in\{1, \ldots, n\}$ such that the leaves $i, j, k$ belong to different components of $L \backslash\{c\}$ (thus $c$ is a vertex of $L$ ) and the minimum of $\left\{c_{i}+a_{i} \cdot P\right\}_{i=1, \ldots, n}$ is attained by the terms corresponding to $i, j, k$.

Let $C=\left\{P_{1}, \ldots, P_{n-2}\right\}$ be a configuration of $n-2$ points in $\mathbb{T P}^{2}$, with $P_{k}=$ $\left(x_{k}, y_{k}, z_{k}\right)$. We say that $C$ is general with respect to $\mathcal{A}$ if $C$ does not lie on a tropical projective curve with support $\mathcal{A} \backslash\left\{a_{i}, a_{j}\right\}$ for any pair $i, j$. This condition can easily be checked as follows. Let $M \in \mathbb{R}^{(n-2) \times n}$ be the matrix with entries $M_{k, \ell}=a_{\ell} \cdot P_{k}=$ $r_{\ell} x_{k}+s_{\ell} y_{k}+t_{\ell} z_{k}$. For any $i, j \in\{1, \ldots, n\}$, let $M^{(i, j)}$ be the maximal minor of $M$ that we get by erasing the $i$ th and $j$ th column of $M$. The tropical determinant of $M^{(i, j)}$ is defined as

$$
\operatorname{tropdet}\left(M^{(i, j)}\right)=\bigoplus_{\sigma \in \mathcal{S}_{i j}}\left(\bigotimes_{k=1}^{n-2} M_{k, \sigma(k)}\right)=\min _{\sigma \in \mathcal{S}_{i j}}\left(\sum_{k=1}^{n-2} a_{\sigma(k)} \cdot P_{k}\right),
$$

where $\mathcal{S}_{i j}$ is the set of bijections $\sigma:\{1, \ldots, n-2\} \rightarrow\{1, \ldots, n\} \backslash\{i, j\}$. Then $C$ is general with respect to $\mathcal{A}$ if and only if each maximal minor $M^{(i, j)}$ is tropically non-singular, i.e. the minimum in $\operatorname{tropdet}\left(M^{(i, j)}\right)$ is attained only once (see [10, Theorem 5.3]).

If $C$ is general, then the set of tropical plane curves with support $\mathcal{A}$ passing through $C$ is a tropical line $L_{C} \subset \mathbb{T P}^{n-1}$ and hence a linear pencil. Note that $L_{C}$ is the intersection of the tropical hypersurfaces $\mathcal{T}\left(\mathcal{H}_{j}\right) \subset \mathbb{T P}^{n-1}$ (i.e. the points $\left(x_{1}, \ldots, x_{n}\right) \in \mathbb{T P}^{n-1}$ where the minimum in $\mathcal{H}_{j}$ is attained at least twice) with $\mathcal{H}_{j}=\min _{i=1, \ldots, n}\left\{a_{i} \cdot P_{j}+x_{i}\right\}$ and $j \in\{1, \ldots, n-2\}$. In case $C$ is not general, this intersection does not need to be a tropical line. Instead we can consider the stable intersection of the tropical hypersurfaces $\mathcal{T}\left(\mathcal{H}_{j}\right)$, where loosely speaking only the transverse intersections of the faces are taken into account. This is a tropical line $L_{C}$ and it is called the stable linear pencil of tropical plane curves through $C$.

An $n$-tree $L$ is said to be compatible with $\mathcal{A}$ if and only if the following condition holds: if a trivalent quartet $(i j \mid k l)$ is a subtree of $L$, then the convex hull of $a_{i}, a_{j}, a_{k}, a_{l}$ has at least one of the segments $\operatorname{conv}\left(a_{i}, a_{j}\right)$ or $\operatorname{conv}\left(a_{k}, a_{l}\right)$ as an edge. 
Note that the combinatorial type of an $n$-tree can be fully recovered by its quartet subtrees. In fact, one of the main problems in phylogenetics is to construct an $n$-tree given a subset of its (quartet) subtrees (see e.g. [1, 6, 11]).

In [10], it is proved that for each configuration $C$ of $n-2$ points the linear pencil $L_{C}$ is compatible with $\mathcal{A}$. We will give a proof of a reverse implication, solving an open question raised in [10].

Theorem 1.2 Let $L$ be a tropical projective line in $\mathbb{T P}^{n-1}$ that is compatible with $\mathcal{A}$. Assume that $L$ is trivalent and that each trivalent vertex $v$ of $L$ corresponds to a maximal triangulation $\Delta(v)$ of $\operatorname{conv}(\mathcal{A})$. Then there exists a general configuration $C$ of $n-2$ points in $\mathbb{T P}^{2}$ such that $L=L_{C}$. In particular, each point in $C$ corresponds to a trivalent vertex of $L$ (in the sense of Proposition 1.1).

Corollary 1.3 For each combinatorial type $\mathcal{T}$ of trivalent $n$-trees that are compatible with $\mathcal{A}$, we can find a general configuration $C$ such that $L_{C}$ is of type $\mathcal{T}$.

For example, if all the points $a_{1}, \ldots, a_{n}$ are on the boundary of $\operatorname{conv}(\mathcal{A})$, then there are precisely $\frac{1}{n-1}\left(\begin{array}{c}2 n-4 \\ n-2\end{array}\right)$ combinatorial types of trivalent trees (out of the $(2 n-5) ! !=$ $1 \cdot 3 \cdot 5 \cdot \ldots \cdot(2 n-5)$ types in total) that are compatible with $\mathcal{A}$ (see [10, Corollary 6.4]) and each type can be obtained by a linear pencil $L_{C}$ with $C$ general.

A short outline of the rest of the paper is as follows. In Sect. 2, we will focus on the fixed locus of tropical linear pencils. In order to show Proposition 1.1, we will give a characterization of tropical lines lying in a tropical hyperplane or one of its skeletons (see Lemma 2.2). In Sect. 3, given a trivalent vertex of an $n$-tree, we will introduce the weaker compatibility condition ( $\mathbf{W}$ ) and show that it implies the existence of a point in the fixed locus (of the second type in Proposition 1.1) of the corresponding tropical linear pencil. For a trivalent $n$-tree, we find a configuration of $n-2$ points in this way. Theorem 1.2 is obtained by proving that this configuration is general.

\section{Fixed Locus of a Linear Pencil}

Example 2.1 Let $\mathcal{A}=\{(0,0,2),(1,0,1),(0,1,1),(1,1,0)\}$. In Fig. 1, four examples of linear pencils of tropical plane curves with support set $\mathcal{A}$ are pictured. The fixed locus of $L_{1}, L_{2}, L_{2}^{\prime}$ and $L_{\infty}$ is respectively $\{(0,0,0)\},\{(0,-1,0),(1,1,0)\}$, $\{(0,0,0),(1,1,0)\}$ and the line segment $[(0,0,0),(0,1,0)]$.

One can see (using Proposition 1.1) that the fixed locus of a linear pencil corresponding to $\mathcal{A}$ has always 1,2 or infinitely many points.

Let $P$ be a point in the fixed locus of a linear pencil $L$ of tropical plane curves with support set $\mathcal{A}$. Then for each $c \in L \subset \mathbb{T} \mathbb{P}^{n-1}$, the minimum of the set

$$
\left\{c_{i}+a_{i} \cdot P\right\}_{i=1, \ldots, n}=\left\{c_{i}+r_{i} x+s_{i} y+t_{i} z\right\}_{i=1, \ldots, n}
$$

is attained at least twice, hence the set $L+\mathcal{A} \cdot P:=\{c+\mathcal{A} \cdot P \mid c \in L\}$ (where $\left.\mathcal{A} \cdot P=\left(a_{i} \cdot P\right)_{i=1, \ldots, n} \in \mathbb{T P}^{n-1}\right)$ is contained in the standard hyperplane $\mathcal{T}\left(x_{1} \oplus\right.$ 

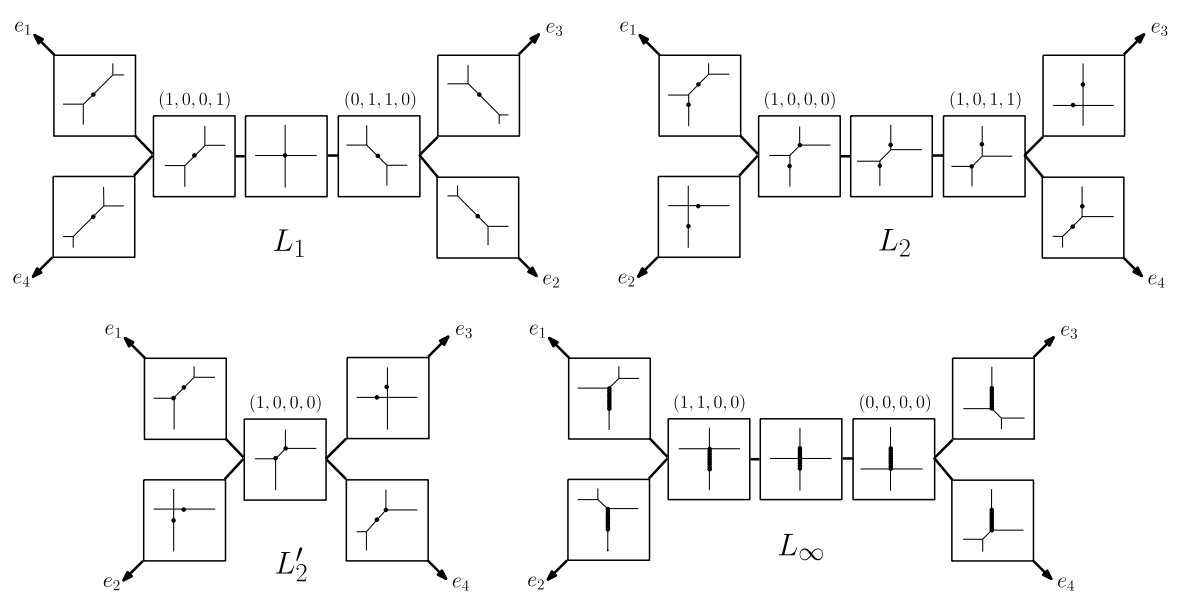

Fig. 1 The linear pencils $L_{1}, L_{2}, L_{2}^{\prime}$ and $L_{\infty}$

$\left.\cdots \oplus x_{n}\right) \subset \mathbb{T P}^{n-1}$. Note that $L+\mathcal{A} \cdot P$ is a translation of $L$, so it is also a tropical line in $\mathbb{T} \mathbb{P}^{n-1}$.

If $t \in\{1, \ldots, n\}$, let $\Pi_{t} \subset \mathbb{T P}^{n-1}$ be the subset consisting of points $\left(x_{1}, \ldots, x_{n}\right)$ such that $\min _{i=1}^{n}\left\{x_{i}\right\}$ is attained at least $t$ times. Note that $\Pi_{t}$ is the $(n-t)$ dimensional skeleton of the standard tropical hyperplane $\Pi_{2}=\mathcal{T}\left(x_{1} \oplus \cdots \oplus x_{n}\right) \subset$ $T \mathbb{P}^{n-1}$ (if $t \geq 2$ ) and that $\Pi_{1}=\mathbb{T P}^{n-1}$. In the following lemma, we examine tropical lines $L$ lying in some $\Pi_{t}$.

Lemma 2.2 Let $\Gamma$ be a tropical line in $\mathbb{T} \mathbb{P}^{n-1}$. If $I \subset\{1, \ldots, n\}$, let

$$
\Pi(I)=\left\{\left(x_{1}, \ldots, x_{n}\right) \in \mathbb{T} \mathbb{P}^{n-1} \mid x_{i}=\min _{j=1}^{n} x_{j} \text { for all } i \in I\right\}
$$

and $\Pi(\Gamma, I)=\Gamma \cap \Pi(I)$.

(a) There exists a point $\pi_{\Gamma} \in \Gamma$ such that $\Pi(\Gamma, I)$ is the union of $\left\{\pi_{\Gamma}\right\}$ with the components of $\Gamma \backslash\left\{\pi_{\Gamma}\right\}$ having no leaf in $I$, for each subset $I \subset\{1, \ldots, n\}$ with $\Pi(\Gamma, I) \neq \emptyset$. Moreover, if $\Gamma \subset \Pi_{t}$ and if $\pi_{\Gamma}$ is an $m$-valent point of $\Gamma$, then $\pi_{\Gamma} \in \Pi_{\left\lceil\frac{m}{m-1} t\right\rceil}$.

(b) Let $p$ be an $m$-valent point of $\Gamma$ and let $C_{1}, \ldots, C_{m}$ be the components of $\Gamma \backslash\{p\}$. Assume that $p \in \Pi(\Gamma, I)$ for some subset $I \subset\{1, \ldots, n\}$ and that $\left|I \backslash I_{j}\right| \geq t$ for all $j \in\{1, \ldots, m\}$, where $t \geq 1$ is an integer and $I_{j}=I \cap C_{j}$. Then we have that $p=\pi_{\Gamma}$ and $\Gamma \subset \Pi_{t}$.

Proof This proof is subdivided into seven steps. Denote by $\Gamma_{I}$ the minimal subtree of $\Gamma$ containing the leaves in $I$.

Step $1 \Gamma_{I} \cap \Pi(\Gamma, I)$ is at most a singleton.

Proof of Step 1 Assume that $x$ and $y$ are two different points in the intersection $\Gamma_{I} \cap \Pi(\Gamma, I)$. Note that this implies that $I$ is not a singleton. We can find $i$ and 
$j$ in $I$ such that the path between the leaves $i$ and $j$ passes through $x$ and $y$ (and assume $x$ is closest to the leaf $i$ ). We can take tropical coordinates such that $x_{i}=y_{i}$ and $x_{j}<y_{j}$. This is in contradiction with the equalities $x_{i}=x_{j}$ and $y_{i}=y_{j}$ since $x, y \in \Pi(\Gamma, I)$.

Step 2 Let $x \in \Pi(\Gamma, I)$ and denote by $\gamma(I) \in \Gamma_{I}$ the point that is closest to $x$. If $y \in \Gamma$ and the path between $y$ and $\gamma(I)$ passes through $x$, then also $y \in \Pi(\Gamma, I)$.

Proof of Step 2 We can take tropical coordinates of $x$ and $y$ such that

$$
\begin{cases}y_{i}=x_{i} & \text { for all } i \in I \\ y_{i}=x_{i}+\epsilon_{i} \text { with } \epsilon_{i} \geq 0 & \text { for all } i \notin I\end{cases}
$$

Then $y_{i}=x_{i}=x_{j}=y_{j}$ for all $i, j \in I$ and $y_{i}=x_{i} \leq x_{j} \leq x_{j}+\epsilon_{j}=y_{j}$ for all $i \in I$ and $j \notin I$, hence $y \in \Pi(\Gamma, I)$.

Step 3 The set $\Pi(\Gamma, I)$ is connected and $\gamma(I) \in \Gamma_{I}$ is independent of $x \in \Pi(\Gamma, I)$.

Proof of Step 3 First we prove that $\gamma(I) \in \Gamma_{I}$ is independent of the choice of the point in $\Pi(\Gamma, I)$. So let $x_{1}, x_{2} \in \Pi(\Gamma, I)$ and denote by $y_{1}$ and $y_{2}$ the points in $\Gamma_{I}$ closest to respectively $x_{1}$ and $x_{2}$. Assume that $y_{1} \neq y_{2}$. There exist elements $i$ and $j$ in $I$ such that $y_{1}$ and $y_{2}$ are contained in the path between the leaves $i$ and $j$ of $\Gamma$ (and assume $y_{1}$ is closest to the leaf $i$ ). We can take tropical coordinates of $x_{1}, x_{2}, y_{1}, y_{2} \in \mathbb{T} \mathbb{P}^{n-1}$ such that

$$
\left(x_{1}\right)_{i}=\left(x_{2}\right)_{i}=\left(y_{1}\right)_{i}=\left(y_{2}\right)_{i}
$$

and

$$
\left(x_{1}\right)_{j}=\left(y_{1}\right)_{j}<\left(x_{2}\right)_{j}=\left(y_{2}\right)_{j} .
$$

This is in contradiction with $x_{1}, x_{2} \in \Pi(\Gamma, I)$.

Now let $x_{1}, x_{2} \in \Pi(\Gamma, I)$. We are going to prove that the path between $x_{1}$ and $x_{2}$ is contained in $\Pi(\Gamma, I)$ as well. For this, it suffices to show that the point $x$ on the path between $x_{1}$ and $x_{2}$ that is closest to $\gamma(I)$ is contained in $\Pi(\Gamma, I)$ (using Step 2). Let $K_{i}$ (for $i \in\{1,2\}$ ) be the leaves in the component of $\Gamma \backslash\{x\}$ containing $x_{i}$ and let $K=\{1, \ldots, n\} \backslash\left(K_{1} \cup K_{2}\right)$. Note that $I \subset K$. We can take tropical coordinates of $x, x_{1}, x_{2}$ such that

$$
\begin{cases}\left(x_{1}\right)_{k}=x_{k} & \text { for all } k \in K \cup K_{2}, \\ \left(x_{1}\right)_{k}=x_{k}+\epsilon_{k} \text { with } \epsilon_{k} \geq 0 & \text { for all } k \in K_{1},\end{cases}
$$

and

$$
\begin{cases}\left(x_{2}\right)_{k}=x_{k} & \text { for all } k \in K \cup K_{1}, \\ \left(x_{2}\right)_{k}=x_{k}+\epsilon_{k}^{\prime} \text { with } \epsilon_{k}^{\prime} \geq 0 & \text { for all } k \in K_{2} .\end{cases}
$$



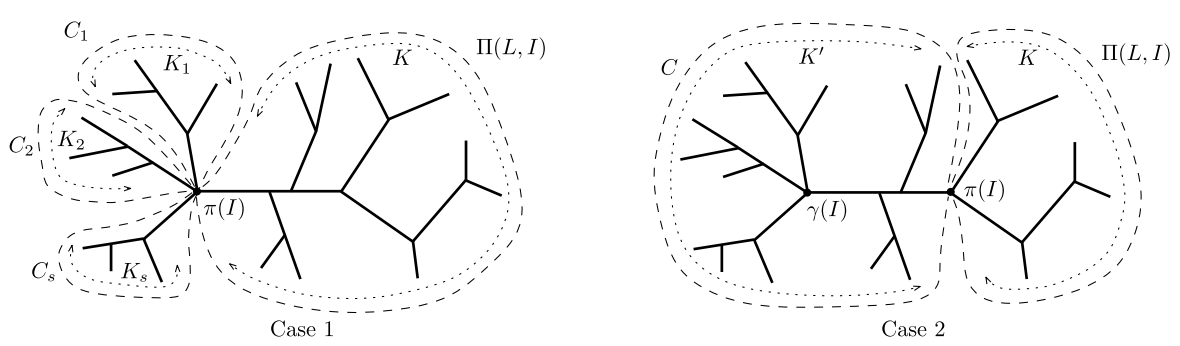

Fig. 2 The two cases in Step 4 of Lemma 2.2

Let $i \in I$. Then we have that

$$
\begin{cases}x_{k}=\left(x_{1}\right)_{k}=\left(x_{2}\right)_{k}=\left(x_{1}\right)_{i}=\left(x_{2}\right)_{i}=x_{i} & \text { if } k \in I \\ x_{k}=\left(x_{1}\right)_{k}=\left(x_{2}\right)_{k} \geq\left(x_{1}\right)_{i}=\left(x_{2}\right)_{i}=x_{i} & \text { if } k \in K \backslash I \\ x_{k}=\left(x_{2}\right)_{k} \geq\left(x_{2}\right)_{i}=x_{i} & \text { if } k \in K_{1} \\ x_{k}=\left(x_{1}\right)_{k} \geq\left(x_{1}\right)_{i}=x_{i} & \text { if } k \in K_{2}\end{cases}
$$

and hence $x \in \bigcap_{i \in I} \Pi(\Gamma,\{i\})=\Pi(\Gamma, I)$.

If $I \subset\{1, \ldots, n\}$ and $\Pi(\Gamma, I)$ is non-empty, we denote by $\pi(I)$ the point in $\Pi(\Gamma, I)$ closest to $\gamma(I)$. The existence of such a point follows from Step 3.

Step 4 If $I^{\prime} \subset I \subset\{1, \ldots, n\}$ with $I^{\prime} \neq \emptyset$ and $\Pi(\Gamma, I) \neq \emptyset$, then we have that $\pi\left(I^{\prime}\right)=\pi(I)$.

Proof of Step 4 First, we give a description of the set $\Pi(\Gamma,\{i\})$ for $i \in I$, where we need to consider the cases $\pi(I)=\gamma(I)$ and $\pi(I) \neq \gamma(I)$ separately (see Fig. 2). Note that $\Pi(\Gamma,\{i\}) \neq \emptyset$ since $\Pi(\Gamma, I) \subset \Pi(\Gamma,\{i\})$.

In the first case (so $\pi(I)=\gamma(I)$ ), let $C_{1}, \ldots, C_{s}$ be the different components of $\Gamma \backslash \Pi(\Gamma, I)$. Let $K_{j}$ be the set of leaves in $C_{j}$ and $K$ the set of leaves in $\Pi(\Gamma, I)$. Note that $I \subset K_{1} \cup \cdots \cup K_{s}, I \cap K_{j} \neq \emptyset$ (by Step 2) and $s>1$ (since $\Gamma_{I}$ is a subtree). Let $i \in K_{j} \cap I$. From Step 2, it follows that

$$
\Pi(\Gamma, I) \cup \bigcup_{r \neq j} C_{r} \subset \Pi(\Gamma,\{i\})
$$

since $\pi(I) \in \Pi(\Gamma,\{i\})$. Assume that this inclusion is strict, hence there exists a point $x \in C_{j}$ such that $x \in \Pi(\Gamma,\{i\})$. Using Steps 2 and 3, we may assume that $x$ is on the path between the leaf $i$ and $\pi(I)$. Take $i^{\prime} \in I$ such that the leaf $i^{\prime}$ is not contained in $K_{j}$, hence $x$ and $\pi(I)$ are contained in $\Gamma_{\left\{i, i^{\prime}\right\}} \cap \Pi\left(\Gamma,\left\{i, i^{\prime}\right\}\right)$, which is in contradiction with Step 1. So we have that $\Pi(\Gamma,\{i\})=\Pi(\Gamma, I) \cup \bigcup_{r \neq j} C_{r}$.

In the second case (so $\pi(I) \neq \gamma(I)$ ), let $C=\Gamma \backslash \Pi(\Gamma, I)$ and denote the set of leaves in $C$ and $\Pi(\Gamma, I)$ by respectively $K^{\prime}$ and $K$. Note that $I \subset K^{\prime}$. If $i \in I$ and $\Pi(\Gamma,\{i\}) \neq \Pi(\Gamma, I)$, consider a point $x \in \Pi(\Gamma,\{i\}) \backslash \Pi(\Gamma, I)$ sufficiently close to $\pi(I)$ (to be precise, let $x$ be an inner point of a finite edge of $\Gamma$ which also contains 
$\pi(I))$. We can take tropical coordinates of $x$ and $\pi(I)$ such that

$$
\begin{cases}\pi(I)_{k}=x_{k} & \text { for all } k \in K^{\prime}, \\ \pi(I)_{k}=x_{k}+\epsilon & \text { for all } k \in K .\end{cases}
$$

If $k \in I$, then $x_{k}=\pi(I)_{k}=\pi(I)_{i}=x_{i}$, hence $x \in \Pi(\Gamma, I)$, a contradiction. Hence, $\Pi(\Gamma,\{i\})=\Pi(\Gamma, I)$.

In both cases, we have that $\pi(\{i\})=\pi(I)$, which implies the equality $\pi\left(I^{\prime}\right)=$ $\pi(I)$ for each non-empty subset $I^{\prime} \subset I$.

Step 5 The point $\pi(I)$ is independent of the set $I \subset\{1, \ldots, n\}$ with $\Pi(\Gamma, I) \neq 0$.

Proof of Step 5 We may assume that $\Gamma \subset \Pi_{t}$ but $\Gamma \not \subset \Pi_{t+1}$ for some $t$. Take $J \subset$ $\{1, \ldots, n\}$ with $|J|=t$ such that $|\Pi(\Gamma, J)|>1$ and such that $\Pi(\Gamma, J) \subset \Pi(\Gamma,\{k\})$ implies $k \in J$. Consider an edge of $\Gamma$ containing $\pi(J)$ that is not fully contained in $\Pi(\Gamma, J)$ and consider points on this edge close to $\pi(J)$ but not in $\Pi(\Gamma, J)$. These points must be contained in some set $\Pi(\Gamma, K)$ with $|K|=t$ and $K \neq J$, so they must be contained in a set $\Pi(\Gamma,\{k\})$ with $k \notin J$. Since the set $\Pi(\Gamma,\{k\})$ is closed, we get that $\pi(J) \in \Pi(\Gamma,\{k\})$. If the leaf $k$ would not be contained in $\Pi(\Gamma, J)$, this would imply that $\Pi(\Gamma,\{k\}) \supset \Pi(\Gamma, J)$ using Step 2, hence $k \in J$, a contradiction. So $k \in \Pi(\Gamma, J)$ and again Step 2 implies $\Gamma=\Pi(\Gamma, J) \cup \Pi(\Gamma,\{k\})$. On the other hand, since

$$
\{\pi(J)\} \subset \Pi(\Gamma, J) \cap \Pi(\Gamma,\{k\})=\Pi(\Gamma, J \cap\{k\}),
$$

we can use Step 4 and see that $\pi(J \cup\{k\})=\pi(J)=\pi(\{k\})$. Now let $I \subset\{1, \ldots, n\}$ be a subset such that $\Pi(\Gamma, I) \neq \emptyset$. Since $\Pi(\Gamma, I \cup J)=\Pi(\Gamma, I) \cap \Pi(\Gamma, J)$ or $\Pi(\Gamma, I \cup\{k\})=\Pi(\Gamma, I) \cap \Pi(\Gamma,\{k\})$ is non-empty, we have that either $\pi(I)=\pi(J)$ or $\pi(I)=\pi(\{k\})$ by Step 4 , hence $\pi(I)=\pi(J)=\pi(\{k\})$.

Step 6 Part (a) of the lemma.

Proof of Step 6 The existence of a point $\pi_{\Gamma}$ follows from Step 5. Now assume that $\pi_{\Gamma}$ is $m$-valent and that $\Gamma \subset \Pi_{t}$. Denote the components of $\Gamma \backslash\left\{\pi_{\Gamma}\right\}$ by $C_{1}, \ldots, C_{m}$. If $r \in\{1, \ldots, m\}$, let $I_{r}$ be the set of leaves $i$ in $C_{r}$ with $\Pi(\Gamma,\{i\}) \neq \emptyset$, and let $\alpha_{r}=\left|I_{r}\right|$. Note that $\pi_{\Gamma} \in \Pi\left(\Gamma, \bigcup_{r=1}^{m} I_{r}\right)$, so it suffices to show that $\alpha_{1}+\cdots+\alpha_{m} \geq \frac{m}{m-1} t$. Since $C_{r} \not \subset \Pi(\Gamma,\{i\})$ for each $i \in I_{r}$, we have that $\left(\alpha_{1}+\cdots+\alpha_{m}\right)-\alpha_{r} \geq t$. Taking the sum of these equations for all $r \in\{1, \ldots, m\}$, we get that $(m-1)\left(\alpha_{1}+\cdots+\alpha_{m}\right) \geq$ $m t$ and the statement follows.

Step 7 Part (b) of the lemma.

Proof of Step 7 Assume that $p \neq \pi_{\Gamma}$ and let $C_{j}$ be the component containing $\pi_{\Gamma}$. Since $\left|I \backslash I_{j}\right| \geq t \geq 1$, it follows that $I \backslash I_{j}$ is non-empty, so we can find an element $i \in$ $I_{k}$ with $k \neq j$. Then (a) implies that $p \notin \Pi(\Gamma,\{i\})$, thus $p \notin \Pi(\Gamma, I)$, a contradiction. In order to show that $\Gamma \subset \Pi_{t}$, note that $C_{j} \cup\{p\} \subset \Pi\left(\Gamma, I \backslash I_{j}\right)$ with $\left|I \backslash I_{j}\right| \geq t$.

We can apply the above lemma in the context of tropical linear pencils. 
Proof of Proposition 1.1 We already showed that $P$ is contained in the fixed locus of $L$ if and only if $\Gamma=L+\mathcal{A} \cdot P$ is contained in the standard tropical hyperplane $\Pi_{2} \subset \mathbb{T} \mathbb{P}^{n-1}$. By Lemma 2.2, this is equivalent to the existence of a point $\pi_{\Gamma} \in \Gamma$ such that one of the following two cases holds: either $\pi_{\Gamma} \in \Pi(\Gamma,\{i, j, k, \ell\})$ where the pairs of leaves $\{i, j\}$ and $\{k, \ell\}$ are contained in different components of $\Gamma \backslash\left\{\pi_{\Gamma}\right\}$, or $\pi_{\Gamma} \in \Pi(\Gamma,\{i, j, k\})$ where the leaves $i, j, k$ are contained in different components of $\Gamma \backslash\left\{\pi_{\Gamma}\right\}$. We conclude that $c=\pi_{\Gamma}-\mathcal{A} \cdot P \in L$ satisfies the conditions of the proposition.

Proposition 2.3 Let $L \subset \mathbb{T} \mathbb{P}^{n-1}$ be the stable linear pencil of tropical plane curves with support set $\mathcal{A}$ through the points $P_{1}, \ldots, P_{n-2} \in \mathbb{T P}^{2}$. Then for each vertex $v$ of $L$ there exists a point $P_{j}$ such that the minimum of $\left\{v_{i}+a_{i} \cdot P_{j}\right\}_{i=1, \ldots, n}$ is attained at least three times.

Proof We know that $L$ is the stable intersection of the tropical hyperplanes

$$
\mathcal{T}\left(\left(a_{1} \cdot P_{i}\right) \otimes x_{1} \oplus \cdots \oplus\left(a_{n} \cdot P_{i}\right) \otimes x_{n}\right) \subset \mathbb{T} \mathbb{P}^{n-1}
$$

Let $v$ be a vertex of $L$. Assume that the minimum of $\left\{v_{i}+a_{i} \cdot P_{j}\right\}_{i=1, \ldots, n}$ is attained precisely two times for each $P_{j}$ and let $\alpha(j), \beta(j) \in\{1, \ldots, n\}$ be the indices of the terms corresponding to the minimum. Then $L$ is locally defined by the stable intersection of the hyperplanes given by $a_{\alpha(j)} \cdot P_{j}+x_{\alpha(j)}=a_{\beta(j)} \cdot P_{j}+x_{\beta(j)}$ in a neighborhood of $v$. Since such an intersection is a linear subspace, we get a contradiction.

So if $L$ is the linear pencil of tropical plane curves through a general configuration of points $P_{i}$, the above result gives some evidence that each $P_{i}$ is situated in case (2) of Proposition 1.1. In fact, we will prove this in the next section.

\section{Compatible Linear Pencils}

Let $L \subset \mathbb{T} \mathbb{P}^{n-1}$ be a tropical line that is compatible with $\mathcal{A}$. A vertex split of $L$ at a trivalent vertex $v \in L$ gives rise to partition $\mathcal{A}_{1}, \mathcal{A}_{2}, \mathcal{A}_{3}$ of $\mathcal{A}$. The compatibility condition implies that for each quartet $a, b, c, d \in \mathcal{A}$ with $a, b \in \mathcal{A}_{i}$ and $c, d \notin \mathcal{A}_{i}$ (with $i \in\{1,2,3\}$ ) holds that the convex hull of the four points $a, b, c, d$ has at least one of the segments $\operatorname{conv}(a, b)$ or $\operatorname{conv}(c, d)$ as an edge. Denote this property on quartets by (W).

Lemma 3.1 Let $\mathcal{A}_{1}, \mathcal{A}_{2}, \mathcal{A}_{3} \subset \mathcal{A}$ be a partition that satisfies (1). Then for each maximal triangulation $\Delta=\left\{T_{1}, \ldots, T_{r}\right\}$ of $\operatorname{conv}(\mathcal{A})$ (with corners of the triangles in $\mathcal{A})$, there exists a triangle $T \in \Delta$ having one vertex in each of the sets $\mathcal{A}_{1}, \mathcal{A}_{2}, \mathcal{A}_{3}$.

Proof First we claim that for each pair $i, j \in\{1,2,3\}$ we have that $\operatorname{conv}\left(\mathcal{A}_{i}\right) \subset$ $\operatorname{conv}\left(\mathcal{A}_{j}\right), \operatorname{conv}\left(\mathcal{A}_{j}\right) \subset \operatorname{conv}\left(\mathcal{A}_{i}\right)$ or $\operatorname{conv}\left(\mathcal{A}_{i}\right) \cap \operatorname{conv}\left(\mathcal{A}_{j}\right)=\emptyset$. Indeed, assume that $\operatorname{conv}\left(\mathcal{A}_{i}\right) \backslash \operatorname{conv}\left(\mathcal{A}_{j}\right), \operatorname{conv}\left(\mathcal{A}_{j}\right) \backslash \operatorname{conv}\left(\mathcal{A}_{i}\right)$ and $\operatorname{conv}\left(\mathcal{A}_{i}\right) \cap \operatorname{conv}\left(\mathcal{A}_{j}\right)$ are non-empty, then there exist elements $a \in \mathcal{A}_{i} \cap \operatorname{conv}\left(\mathcal{A}_{j}\right)$ and $b \in \mathcal{A}_{i} \backslash \operatorname{conv}\left(\mathcal{A}_{j}\right)$. The line segment 


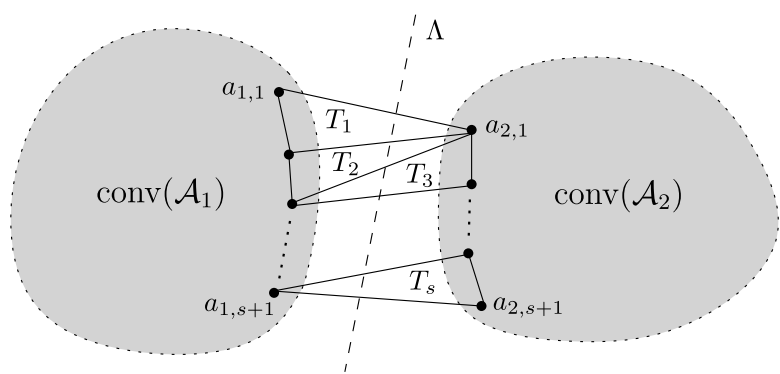

$$
\begin{gathered}
T_{1}=\operatorname{conv}\left(a_{1,1}, a_{1,2}, a_{2,1}\right) \\
T_{2}=\operatorname{conv}\left(a_{1,2}, a_{1,3}, a_{2,2}\right) \\
T_{3}=\operatorname{conv}\left(a_{1,3}, a_{2,3}, a_{2,4}\right) \\
\vdots \\
T_{s}=\operatorname{conv}\left(a_{1, s}, a_{2, s}, a_{2, s+1}\right)
\end{gathered}
$$

Fig. 3 First configuration

$\operatorname{conv}(a, b)$ cuts the border of $\operatorname{conv}\left(\mathcal{A}_{j}\right)$ at a line segment $\operatorname{conv}(c, d)$ with $c, d \in \mathcal{A}_{j}$. The convex hull of the quartet $a, b, c, d \in \mathcal{A}$ has $\operatorname{conv}(a, b)$ or $\operatorname{conv}(c, d)$ as diagonals, a contradiction. Using the above claim, there are three possible configurations of $\mathcal{A}_{1}, \mathcal{A}_{2}, \mathcal{A}_{3}$ (after a renumbering if necessary): $\operatorname{conv}\left(\mathcal{A}_{1}\right), \operatorname{conv}\left(\mathcal{A}_{2}\right), \operatorname{conv}\left(\mathcal{A}_{3}\right)$ pairwise disjoint, $\left(\operatorname{conv}\left(\mathcal{A}_{1}\right) \cup \operatorname{conv}\left(\mathcal{A}_{2}\right)\right) \subset \operatorname{conv}\left(\mathcal{A}_{3}\right)$ or $\operatorname{conv}\left(\mathcal{A}_{1}\right) \subset \operatorname{conv}\left(\mathcal{A}_{2}\right) \subset$ $\operatorname{conv}\left(\mathcal{A}_{3}\right)$. The latter configuration is impossible. Indeed, take $c \in \mathcal{A}_{1}$ and $d \in$ $\mathcal{A}_{3} \backslash \operatorname{conv}\left(\mathcal{A}_{2}\right)$. Then the line segment $\operatorname{conv}(c, d)$ passes through a edge $\operatorname{conv}(a, b)$ of the border of $\operatorname{conv}\left(\mathcal{A}_{2}\right)$ and the quartet $a, b, c, d$ is in contradiction with ( $\left.\mathbb{A}^{-}\right)$.

Assume we are in the case of the first configuration, so the $\operatorname{sets} \operatorname{conv}\left(\mathcal{A}_{i}\right)$ are pairwise disjoint. Since $\Delta$ is a triangulation of $\operatorname{conv}(\mathcal{A})$ (and thus not only of the sets $\operatorname{conv}\left(\mathcal{A}_{i}\right)$ ), we may assume (after a renumbering if necessary) there is an edge of a triangle $T \in \Delta$ connecting a point in $\mathcal{A}_{1}$ with a point in $\mathcal{A}_{2}$. Let $\Delta^{\prime} \subset \Delta$ be the set of all triangles having two vertices in $\mathcal{A}_{1}$ and one vertex in $\mathcal{A}_{2}$ or vice versa. If $\Delta^{\prime}=\emptyset$, then the third vertex of the triangle $T$ is contained in $\mathcal{A}_{3}$ and the statement follows. Now assume $\Delta^{\prime}$ is non-empty, say $\Delta^{\prime}=\left\{T_{1}, \ldots, T_{s}\right\}$ (after a renumbering if necessary). We can draw a line $\Lambda$ subdividing the plane into two half-planes $H_{1}$ and $H_{2}$ such that $\operatorname{conv}\left(\mathcal{A}_{1}\right) \subset H_{1}, \operatorname{conv}\left(\mathcal{A}_{2}\right) \subset H_{2}$ and $\Lambda \cap \mathcal{A}=\emptyset$. Using an identification of the line $\Lambda$ with $\mathbb{R}$ (using an affine coordinate), each of the triangles $T_{i} \in \Delta^{\prime}$ cuts $\Lambda$ in a closed interval $I_{i}$ of $\mathbb{R}$ and two intervals $I_{i}$ and $I_{j}$ (with $i, j \in\{1, \ldots, s\}$ different) are either pairwise disjoint or subsequent (i.e. the intersection is a point). We may assume that $T_{1}, \ldots, T_{s}$ are numbered in such a way that $\max \left(I_{i}\right) \leq \min \left(I_{i+1}\right)$ for all $i \in\{1, \ldots, s-1\}$. If one of the above inequalities is strict, then the statement follows, since the edge of $T_{i}$ containing $\max \left(I_{i}\right)$ has to be the edge of another triangle $T \in \Delta$ having its third vertex in $\mathcal{A}_{3}$. Hence the intervals $I_{1}, \ldots, I_{s}$ are subsequent. Denote by $a_{1, i} \in \mathcal{A}_{1}$ and $a_{2, i} \in \mathcal{A}_{2}$ the two vertices of the edge of $T_{i}$ containing $\min \left(I_{i}\right) \in \Lambda$ and by $a_{1, s+1} \in \mathcal{A}_{1}$ and $a_{2, s+1} \in \mathcal{A}_{2}$ the two vertices of the edge of $T_{s}$ containing $\max \left(I_{s}\right)$ (see Fig. 3). Note that either $a_{1, i}=a_{1, i+1}$ and $T_{i}=\operatorname{conv}\left(a_{1, i}, a_{2, i}, a_{2, i+1}\right)$ or $a_{2, i}=a_{2, i+1}$ and $T_{i}=\operatorname{conv}\left(a_{1, i}, a_{1, i+1}, a_{2, i}\right)$. If $\operatorname{conv}\left(a_{1,1}, a_{2,1}\right)$ (resp. $\left.\operatorname{conv}\left(a_{1, s+1}, a_{2, s+1}\right)\right)$ is not a part of the border of $\operatorname{conv}(\mathcal{A})$, then this line segment is the edge of a triangle $T \in \Delta$ different from $T_{1}$ (resp. $T_{S}$ ) with one vertex in each of the three sets $\mathcal{A}_{i}$. So we may assume that $\operatorname{conv}\left(a_{1,1}, a_{2,1}\right)$ and $\operatorname{conv}\left(a_{1, s+1}, a_{2, s+1}\right)$ are parts of the border of $\operatorname{conv}(\mathcal{A})$. 
Fig. 4 Second configuration

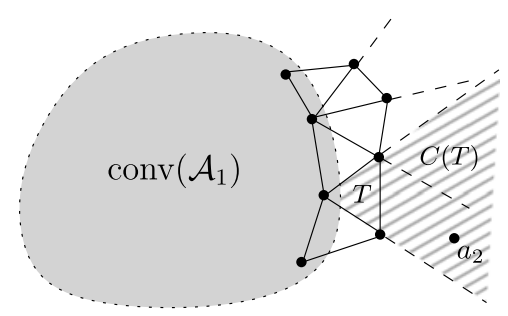

First note that $\operatorname{conv}\left(a_{1,1}, a_{1, s+1}, a_{2,1}, a_{2, s+1}\right)$ does not contain a point of $\mathcal{A}_{3}$ since

$$
\begin{aligned}
& \operatorname{conv}\left(a_{1,1}, a_{1, s+1}, a_{2,1}, a_{2, s+1}\right) \\
& \subset \operatorname{conv}\left(a_{1,1}, \ldots, a_{1, s+1}, a_{2, i}, \ldots, a_{2, s+1}\right) \\
& \subset \operatorname{conv}\left(a_{1,1}, \ldots, a_{1, s+1}\right) \cup \operatorname{conv}\left(a_{2,1}, \ldots, a_{2, s+1}\right) \cup \bigcup_{i=1}^{s} T_{i} \\
& \subset \operatorname{conv}\left(\mathcal{A}_{1}\right) \cup \operatorname{conv}\left(\mathcal{A}_{2}\right) \cup \bigcup_{i=1}^{s} T_{i} .
\end{aligned}
$$

Let $a_{3} \in \mathcal{A}_{3}$. We may assume that $a_{3}$ is contained in the half-plane $H_{1}$. If $a_{1,1}=$ $a_{1, s+1}$, then $a_{3}$ is contained in $\operatorname{conv}\left(a_{1,1}, a_{2,1}, a_{2, s+1}\right)$ since $H_{1} \cap \operatorname{conv}(\mathcal{A})=H_{1} \cap$ $\operatorname{conv}\left(a_{1,1}, a_{2,1}, a_{2, s+1}\right)$, a contradiction. So we have that $a_{1,1} \neq a_{1, s+1}$ and the quartet $a_{1,1}, a_{1, s+1}, a_{2,1}, a_{3}$ is in contradiction with (4).

To finish the proof, we have to take care of the second configuration, so $\left(\operatorname{conv}\left(\mathcal{A}_{1}\right) \cup \operatorname{conv}\left(\mathcal{A}_{2}\right)\right) \subset \operatorname{conv}\left(\mathcal{A}_{3}\right)$. Assume there is no triangle $T \in \Delta$ having one vertex in $\mathcal{A}_{1}$ and one in $\mathcal{A}_{2}$. For $(i, j) \in\{(3,0),(2,1),(1,2)\}$, denote by $\Delta_{i, j} \subset \Delta$ the set of all triangles $T$ having $i$ vertices in $\mathcal{A}_{1}$ and $j$ vertices in $\mathcal{A}_{3}$. For a triangle $T=\operatorname{conv}\left(a_{1}, a_{3}, a_{3}^{\prime}\right) \in \Delta_{1,2}$ with $a_{1} \in \mathcal{A}_{1}$ and $a_{3}, a_{3}^{\prime} \in \mathcal{A}_{3}$, let $C(T) \subset \mathbb{R}^{2}$ be the cone with top $a_{1}$ over the line segment $\left[a_{3}, a_{3}^{\prime}\right]$ (see Fig. 4). Then we can see that

$$
\left(\mathbb{R}^{2} \backslash \bigcup_{T \in \Delta_{1,2}} C(T)\right) \subset\left(\bigcup_{T \in \Delta_{3,0}} T \cup \bigcup_{T \in \Delta_{2,1}} T\right) \subset\left(\operatorname{conv} \mathcal{A}_{1} \cup \bigcup_{T \in \Delta_{2,1}} T\right)
$$

If $a_{2} \in \mathcal{A}_{2}$, then $a_{2} \notin \operatorname{conv}\left(\mathcal{A}_{1}\right) \cup \bigcup_{T \in \Delta_{2,1}} T$ and thus $a_{2} \in C(T)$ for some triangle $T=\operatorname{conv}\left(a_{1}, a_{3}, a_{3}^{\prime}\right) \in \Delta_{1,2}$. Hence the quartet of points $a_{1}, a_{2}, a_{3}, a_{3}^{\prime}$ is in contradiction with ( $\left.\mathbb{A}^{\prime}\right)$. So there exists a triangle $T \in \Delta$ connecting $\mathcal{A}_{1}$ with $\mathcal{A}_{2}$ and by using analogous arguments as in the case of the first configuration, we can prove the existence of a triangle $T \in \Delta$ having one vertex in each of the sets $\mathcal{A}_{1}, \mathcal{A}_{2}, \mathcal{A}_{3}$.

Proposition 3.2 Let $L \subset \mathbb{T P}^{n-1}$ be a linear pencil of tropical plane curves with support $\mathcal{A}$ such that $L$ is compatible with $\mathcal{A}$. Let $v$ be a trivalent vertex of $L$ such that $\Delta(v)$ is a maximal triangulation. Then $v$ corresponds to a point $P_{v}$ in the fixed locus of $L$.

Proof By Lemma 3.1, we find a triangle $T_{v}=\operatorname{conv}\left(a_{i}, a_{j}, a_{k}\right)$ of the maximal subdivision $\Delta(v)$ of $\mathcal{A}$ such that $a_{i} \in \mathcal{A}_{1}, a_{j} \in \mathcal{A}_{2}$ and $a_{k} \in \mathcal{A}_{3}$. Let $P_{v}=(x, y, z) \in$ $\mathcal{T}\left(F_{v}\right) \subset \mathbb{T P}^{2}$ be the point which is dual to the triangle $T_{v}$, hence 
Fig. 5 The subtree $\Lambda_{w}$ of $L$

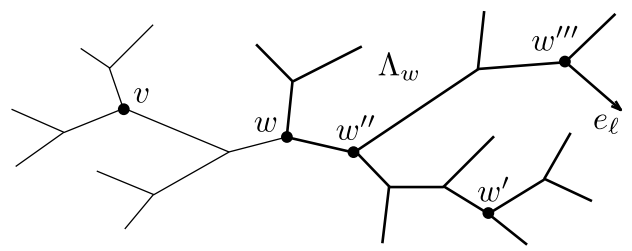

$$
\begin{aligned}
v_{i}+r_{i} x+s_{i} y+t_{i} z & =v_{j}+r_{j} x+s_{j} y+t_{j} z \\
& =v_{k}+r_{k} x+s_{k} y+t_{k} z<v_{\ell}+r_{\ell} x+s_{\ell} y+t_{\ell} z
\end{aligned}
$$

for all $\ell \in\{1, \ldots, n\} \backslash\{i, j, k\}$. From Proposition 1.1, it follows that $P_{v}$ is contained in the fixed locus of $L$.

Let $L \subset \mathbb{T} \mathbb{P}^{n-1}$ be a linear pencil of tropical plane curves with support $\mathcal{A}$. Assume that $L$ is compatible with $\mathcal{A}$, trivalent and that each (trivalent) vertex of $L$ gives rise to a maximal triangulation of $\operatorname{conv}(\mathcal{A})$. Note that Proposition 3.2 implies that the fixed locus of $L$ contains a configuration of $n-2$ points and in order to prove Theorem 1.2, we need to show that this configuration is general.

We are going to assign a subgraph $G_{v}$ of $K_{n-2, n}$ to each vertex $v$ of $L$. We identify the vertices of $K_{n-2, n}$ with the vertices of $L$ (of which there are $n-2$ ) and the elements of $\mathcal{A}$ (of which there are $n$ ). The edge $\left(w, a_{\ell}\right)$ of $K_{n-2, n}$ is an edge of $G_{v}$ if and only if $a_{\ell}$ is a corner of $T_{w}$ and the path between the leaf $\ell$ and $v$ passes through $w$. So $\left(w, a_{\ell}\right) \in E\left(G_{v}\right)$ if and only if $v_{\ell}+a_{\ell} \cdot P_{w}=\min _{k=1, \ldots, n}\left\{v_{k}+a_{k} \cdot P_{w}\right\}$. Note that precisely two of the three corners of $T_{w}$ satisfy this condition for $w \neq v$, and all three for $w=v$. If $a_{\ell} \in \mathcal{A}$, the vertex $w$ of $L$ that is closest to the leaf $\ell$ gives rise to an edge $\left(w, a_{\ell}\right) \in E\left(G_{v}\right)$. We conclude that $\left|E\left(G_{v}\right)\right|=2(n-3)+3=2 n-3$ and $\left|V\left(G_{v}\right)\right|=n+(n-2)=2 n-2$.

Lemma 3.3 The graph $G_{v}$ is connected and has no cycles.

Proof First note that, in order to prove that $G_{v}$ is connected, it suffices to show that each vertex $w \in V\left(G_{v}\right)$ is connected with $v$. Let $\Lambda_{w}$ be the union of $\{w\}$ with the components of $L \backslash\{w\}$ not containing $v$. We will prove by induction on the number $\lambda_{w}$ of vertices in the subtree $\Lambda_{w}$ that each vertex $w^{\prime}$ in $\lambda_{w}$ is connected with $w$ in $G_{v}$. Since $\Lambda_{v}=L$, this would imply the connectedness. If $\lambda_{w}=1$, there is nothing to prove. Now assume the statement is proven for any subtree $\Lambda_{w}$ with $\lambda_{w}<\lambda$ and take a subtree $\Lambda_{w}$ with $\lambda_{w}=\lambda$. Let $w^{\prime} \neq w$ be a vertex in $\Lambda$. Consider the vertex $w^{\prime \prime} \neq w$ closest to $w$ on the path between $w$ and $w^{\prime}$ (so $w^{\prime} \in \Lambda_{w^{\prime \prime}}$ ) and take the corner $a_{\ell}$ of $T_{w}$ corresponding to a leaf $\ell$ lying in $\Lambda_{w^{\prime \prime}}$. Denote by $w^{\prime \prime \prime}$ the vertex of $\Lambda_{w^{\prime \prime}} \subset L$ which is closest to $\ell$. Then $\left(w^{\prime \prime \prime}, a_{\ell}\right),\left(w, a_{\ell}\right) \in E\left(G_{v}\right)$, hence $w$ and $w^{\prime \prime \prime}$ are connected in $G_{v}$. On the other hand, by the induction hypothesis on the subtree $\Lambda_{w^{\prime \prime}}$, both $w^{\prime}$ and $w^{\prime \prime \prime}$ are connected with $w^{\prime \prime}$ (Fig. 5). We conclude that $w$ is connected with $w^{\prime}$ in $G_{v}$.

Since $G_{v}$ is connected, we can compute the genus (or first Betti number) of $G_{v}$ as $g\left(G_{v}\right)=\left|E\left(G_{v}\right)\right|-\left|V\left(G_{v}\right)\right|+1=0$. This implies that $G_{v}$ has no cycles.

In the same way as we introduced $G_{v} \subset K_{n-2, n}$ for vertices $v$ of $L$, we can define $G_{c} \subset K_{n-2, n}$ for 2-valent points $c$ of $L$, i.e. $\left(w, a_{\ell}\right) \in E\left(G_{c}\right)$ if and only if $c_{\ell}+a_{\ell}$. 
$P_{w}=\min _{k=1, \ldots, n}\left\{c_{k}+a_{k} \cdot P_{w}\right\}$. Note that if $c$ is contained in an edge adjacent to $v$, then $V\left(G_{c}\right)=V\left(G_{v}\right)$ and $E\left(G_{c}\right)=E\left(G_{v}\right) \backslash\left\{\left(v, a_{\ell}\right)\right\}$ where $a_{\ell}$ is the corner of $T_{v}$ that corresponds to the leaf $\ell$ of $L$ lying on the same side of $v$ as $c$. This implies that $G_{c}$ has two connected components of genus 0 . If $\mathcal{B} \subset \mathcal{A}$, denote by $N(\mathcal{B})$ the neighborhood of $\mathcal{B}$ in $G_{c}$, i.e. the set of vertices $v$ of $L$ such that $\left(v, a_{\ell}\right) \in E\left(G_{c}\right)$ for some $a_{\ell} \in \mathcal{B}$.

Lemma 3.4 Let $i, j \in\{1, \ldots, n\}$ be leaves of $L$ and $c$ be a 2-valent point of $L$ on the path between $i$ and $j$. Then the inequality $|N(\mathcal{B})| \geq|\mathcal{B}|$ holds for each subset $\mathcal{B} \subset \mathcal{A} \backslash\left\{a_{i}, a_{j}\right\}$.

Proof Let $C_{i}$ and $C_{j}$ be the components of $L \backslash\{c\}$ such that $i \in C_{i}$ and $j \in C_{j}$. We are going to prove this lemma by induction on $|\mathcal{B}|$. If $\mathcal{B}=\emptyset$, there is nothing to prove. Now assume that $\mathcal{B} \neq \emptyset$ and let $\mathcal{B}_{i}=\mathcal{B} \cap C_{i}$ and $\mathcal{B}_{j}=\mathcal{B} \cap C_{j}$. Since $\mathcal{B}=\mathcal{B}_{i} \cup \mathcal{B}_{j}$, we may assume that $\mathcal{B}_{i} \neq \emptyset$. Denote by $w$ the end point of the edge that contains $c$ and that is contained in $C_{i}$. For each vertex or leaf $v$ of $C_{i}$ different from $w$, write $\rho(v) \neq v$ to denote the vertex of $L$ on the path between $v$ and $c$ closest to $v$. Let $\mathcal{V}$ be the set of vertices or leaves $v$ of $C_{i}$ such that all leaves for which the path to $c$ passes through $v$ are contained in $\mathcal{B}_{i}$ and such that this is not the case for $\rho(v)$. We can take an element $v \in \mathcal{V}$ such that the component of $L \backslash\{\rho(v)\}$ not containing $c$ and $v$ does not have any leaf in $\mathcal{B}_{i}$. Note that the triangle $T_{\rho(v)}$ has precisely one corner $a_{\ell} \in \mathcal{B}_{i}$ and that $\left(\rho(v), a_{\ell}\right) \in E\left(G_{c}\right)$, hence $\rho(v) \in N(\mathcal{B})$. On the other hand, we see that $\rho(v) \notin N\left(\mathcal{B} \backslash\left\{a_{\ell}\right\}\right)$, so the induction hypothesis implies that

$$
|N(\mathcal{B})| \geq\left|N\left(\mathcal{B} \backslash\left\{a_{\ell}\right\}\right)\right|+1 \geq\left|\mathcal{B} \backslash\left\{a_{\ell}\right\}\right|+1=|\mathcal{B}| .
$$

Proof of Theorem 1.2 Since $L$ is a trivalent tree with $n$ leaves, it has precisely $n-$ 2 trivalent vertices which we will denote by $v_{1}, \ldots, v_{n-2}$. Using Proposition 3.2, each $v_{i}$ gives rise to a point $P_{i}$ in the fixed locus of $L$. It suffices to show that the configuration $C=\left\{P_{1}, \ldots, P_{n-2}\right\}$ is general with respect to $\mathcal{A}$. Therefore, we need to show that each maximal minor $M^{(i, j)}$ of the matrix $M$ with $M_{k, \ell}=a_{\ell} \cdot P_{k}$ is tropically non-singular.

Let $c$ be a 2-valent point of $L$ on the path between $i$ and $j$ and consider the graph $G_{c} \subset K_{n-2, n}$. Hall's Marriage Theorem (see [7]) and Lemma 3.4 imply that there is a matching in $G_{c}$ between $\left\{v_{1}, \ldots, v_{n-2}\right\}$ and $\mathcal{A} \backslash\left\{a_{i}, a_{j}\right\}$. Note that this matching is unique since $G_{c}$ contains no cycles. Let $\psi$ be the bijection in $\mathcal{S}_{i j}$ that corresponds to the matching. We claim that the minimum in $\operatorname{tropdet}\left(M^{(i, j)}\right)$ is attained only by the term that corresponds to $\psi$. Indeed, let $\sigma \in S_{i j}$ be different from $\psi$. For all $k \in$ $\{1, \ldots, n-2\}$, we have that $c_{\sigma(k)}+a_{\sigma(k)} \cdot P_{k} \geq c_{\psi(k)}+a_{\psi(k)} \cdot P_{k}$ and equality holds if and only if $\left(v_{k}, a_{\sigma(k)}\right) \in E\left(G_{c}\right)$. If we take the sum of all these inequalities (and erase the term $\left.\sum_{\ell \in\{1, \ldots, n\} \backslash\{i, j\}} c_{\ell}\right)$, we see that

$$
\sum_{k=1}^{n-2} a_{\sigma(k)} \cdot P_{k}>\sum_{k=1}^{n-2} a_{\psi(k)} \cdot P_{k},
$$

since at least one of the inequalities must be strict. This proves the claim and the theorem. 
Remark 3.5 The linear pencils $L_{2}^{\prime}$ and $L_{\infty}$ in Example 2.1 show that the two extra conditions on $L$ in Theorem 1.2, i.e. that $L$ is trivalent and each vertex gives rise to a maximal subdivision of $\operatorname{conv}(\mathcal{A})$, are necessary. Note that $L_{2}^{\prime}$ and $L_{\infty}$ are the stable linear pencils corresponding to respectively $\{(0,0,0),(1,1,0)\}$ and $\{(0,0,0),(0,1,0)\}$. In fact, both linear pencils can be seen as a limit case of linear pencils satisfying the two conditions and thus corresponding to general configurations, but the limit of these configurations is non-general.

Proof of Corollary 1.3 Let $\Delta$ be a maximal triangulation of $\operatorname{conv}(\mathcal{A})$ and consider the cone of the secondary fan (see [3]) corresponding to $\Delta$, i.e. the set of all points $\left(c_{1}, \ldots, c_{n}\right) \in \mathbb{R}^{n}$ such that the projection to the last coordinate of the lower faces of the polytope

$$
\operatorname{conv}\left(\left(a_{1}, c_{1}\right), \ldots,\left(a_{n}, c_{n}\right)\right) \subset \mathbb{R}^{4}
$$

is $\Delta$. We can take a tree $L \subset \mathbb{T} \mathbb{P}^{n-1}$ of type $\mathcal{T}$ such that all its finite edges are contained in this cone (by taking the edge lengths small enough). Now the statement follows from Theorem 1.2.

\section{References}

1. Böcker, S., Dress, A.W.M., Steel, M.: Patching up X-trees. Ann. Comb. 3, 1-12 (1999)

2. Brodsky, S., Sturmfels, B.: Tropical quadrics through three points. Linear Algebra Appl. 435(7), 1778-1785 (2011)

3. De Loera, J.A., Rambau, J., Santos, F.: Triangulations: Structures for Algorithms and Applications. Algorithms and Computation in Mathematics, vol. 25 (2010) 539 p

4. Einsiedler, M., Kapranov, M., Lind, D.: Non-Archimedean amoebas and tropical varieties. J. Reine Angew. Math. 601, 139-157 (2006)

5. Gathmann, A., Markwig, H.: The Caporaso-Harris formula and plane relative Gromov-Witten invariants in tropical geometry. Math. Ann. 338, 845-868 (2007)

6. Grunewald, S., Huber, K.T., Moulton, V., Semple, C.: Encoding phylogenetic trees in terms of weighted quartets. J. Math. Biol. 56, 465-477 (2008)

7. Hall, P.: On representatives of subsets. J. Lond. Math. Soc. 10, 26-30 (1935)

8. Mikhalkin, G.: Enumerative tropical algebraic geometry in $\mathbb{R}^{2}$. J. Am. Math. Soc. 18(2), 313-377 (2005)

9. Payne, S.: Fibers of tropicalization. Math. Z. 262, 301-311 (2009)

10. Richter-Gebert, J., Sturmfels, B., Theobald, T.: First steps in tropical geometry. In: Idempotent Mathematics and Mathematical Physics, Contemp. Math., vol. 377, pp. 289-317. Amer. Math. Soc, Providence (2005)

11. Semple, C., Steel, M.: Tree reconstruction from multi-state characters. Adv. Appl. Math. 28(2), 169184 (2002)

12. Speyer, D., Sturmfels, B.: The tropical Grassmannian. Adv. Geom. 4, 389-411 (2004) 